\title{
Aesthetic
Plastic
Surgery
}

(C) 2003 Springer-Verlag New York Inc

\section{Liposuction in Benign Symmetric Lipomatosis: Sense or Senseless?}

\author{
Nicolas A.C. Verhelle, ${ }^{1,2}$ Jean-Luc Nizet, ${ }^{2}$ Bert Van den hof, ${ }^{1}$ Paul Guelinckx, ${ }^{2}$ and Olivier Heymans ${ }^{2}$ \\ ${ }^{1}$ Leuven, Belgium \\ ${ }^{2}$ Liège, Belgium
}

\begin{abstract}
Benign symmetric lipomatosis is a rare form of typical fat distribution in the shoulders, the arms, and the neck that can compromise the respiratory, nutritional, and psychological status of the patient. Alcoholism, malignant tumors of the upper airways, endocrine tumors, hypothyroidism, diabetes, and hypertriglyceridemia are often associated with its occurrence. Surgical removal via lipectomy or liposuction can give good cosmetic results, although recurrences often occur. Liposuction has become the first choice to treat this disorder in patients with smaller masses. We have operated on four such cases in which liposuction failed and surgical excision had to be performed. We present a summary of the clinical characteristics of all four patients and discuss the different treatment options.
\end{abstract}

Key words: Madelung's disease-Failed liposuction

Benign symmetric lipomatosis (BSL) was first described by Brodie in 1846, but from 1888 it has also been known as Madelung's disease or maladie de Lenois-Bensaude [6,21].

The etiology is still controversial. It seems to be more frequent in countries around the Mediterranean Sea. The male to female ratio has been reported as 15 to 1 [2]. Although alcoholism is often associated, malignant tumors of the upper airways, endocrine tumors, hypothyroidism, diabetes, and hypertriglyceridemia are also commonly found. However, none of these disorders has a causal relationship with BSL. Most recent hypotheses acknowledge BSL as an expression of an alcohol-induced endocrine disorder [4], and biochemical studies on muscle and adipose cells

Correspondence to Nicolas A.C. Verhelle, Department of Maxillo-Facial and Plastic Surgery, CHU Sart Tilman, 4000 Liége, Belgium have shown evidence of mitochondrial respiratory enzyme dysfunction, which is closely related to lipid metabolism [6]. The pathognomic signs are a symmetric distribution of the adipose tissue in the submandibular region, over the suboccipital area, the shoulder, and proximal upper extremities, in contrast to the distal upper extremities and body. These are rather asthenic, resulting in a pseudo-athletic appearance.

The clinical course of benign symmetric lipomatosis is also characteristic: a rapidly progressing growth of the fat tissue during the early phases of the disease, which thereafter either slowly progresses or remains stable for many years. Regression of the subcutaneous mass occasionally occurs, but complete involution has never been described [2,5,8]. Malignant degeneration of the fat tissue into myxoid liposarcoma has been reported [7]. Due to frequent inframandibular location of the deposits, aesthetic or psychological problems and compression of the trachea or mediastinum are described.

\section{Patients and Methods}

We operated five patients - four males and one female - with a mean age 52.5 years (range, 42-61). All had a history of alcohol abuse, and one male had diabetes mellitus. They presented with swelling of subcutaneous fatty tissue in the submandibular region and in the upper aspect of the back (Fig. 1). These masses existed on an average of four years (range, 2-7), and all patients consulted because of cosmetic reasons. On palpation, this subcutaneous fat was firm and elastic. The overlying skin was tense and in three patients telangiectasias were present. In four patients, laboratory investigations revealed elevated serum hepatic levels (67-540 U/L) and elevation of 


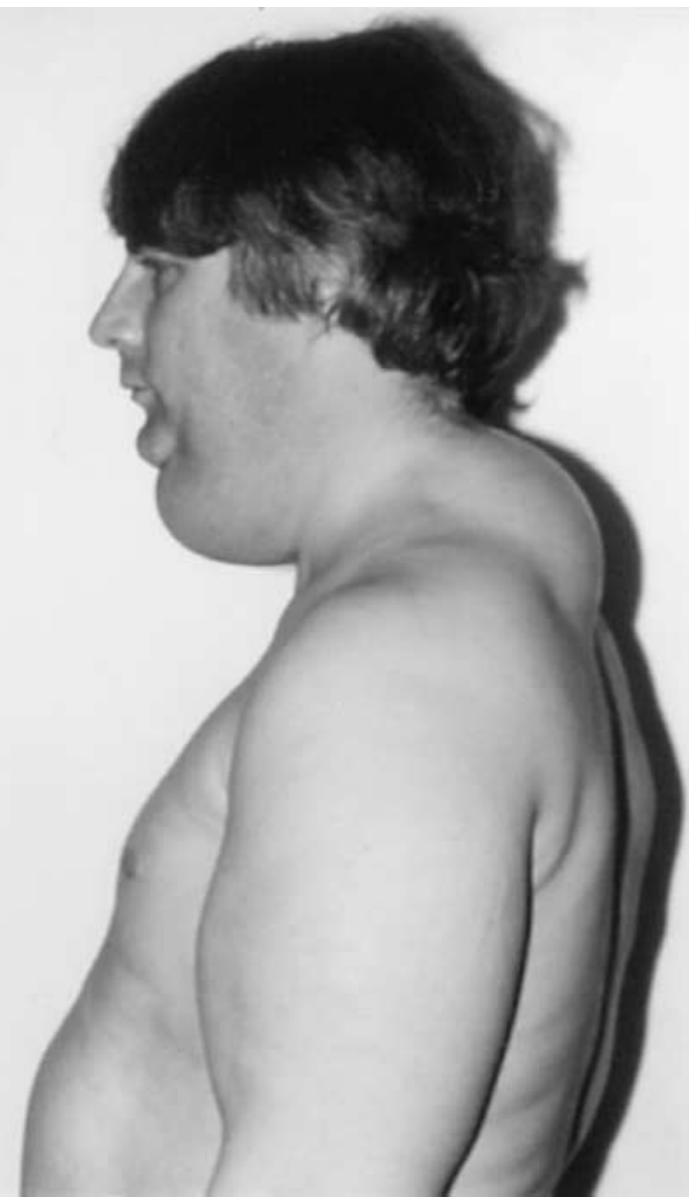

Fig. 1. Swelling of subcutaneous fatty tissue in the submandibular region and in the upper aspect of the back.

cholesterol and triglycerides. Chest x-ray revealed no abnormalities and abdominal ultrasound showed hepatomegaly in two males. MRI was performed two patients and showed a symmetric swelling of the subcutaneous fat tissue. Two males asked us to remove the subcutaneous fat of the submandibular region and upper aspect of the back, while the others only wanted a removal of the fat at the back. We started our treatment with liposuction after infiltration, as is the recommended approach for this kind of disease. Due to the firmness and slow progress of aspiration, especially for the back location, we stopped this procedure and continued with surgical excision. In all five cases we used a W-shaped incision (Fig. 2) between the scapulae and a prolonged thyroidectomy incision for removal of the submandibular fat. The excised tissue was firm in consistency and dryer than normal subcutaneous fat. The distribution was diffuse without encapsulation. Tissue samples were taken for histology examination. The postoperative course was uneventful and the mean hospital stay was three days (range, 2-4).Histology revealed normal mature fat tissue with a normal lobulated structure. The masses contained some fibrous septa, but no abnormal changes were found. The mean postoperative follow-up was 26 months (range, 10 48) and in all patients the subcutaneous swelling remained relatively constant, although we note a recurrence in one male. As of now, no secondary procedures have been performed.

\section{Discussion}

The diagnosis of BSL is clinical and can be based on a typical fat distribution in the shoulders, the arms, and the neck. Histologically, it appears as normal, mature, subcutaneous fatty tissue divided by fibrous septa - not encapsulated - with extensions into surrounding structures, and without pathognomic changes. The adipocytes have a normal aspect, and are not hypertrophic. The neoplastic increase of the fatty tissue is assumed to result from hyperplasia. Differential diagnosis includes a variety of other rare diseases such as lymphoproliferative diseases, lymph node enlargement, enlarged goiter, cervical cysts, and benign tumors. They can be differentiated by computer tomography or biopsy. Various approaches for treatment exist. Nonsurgical treatment includes alcohol abstinence as the first step, although this has no effect on its own $[1,8,10]$. However, the search for and treatment of associated diseases is essential. Dietary treatment and weight loss have no effect on this type of lipomatous tissue. Temporary results are described with thyroid extracts and chymotrypsin injections $[1,2,5]$. Surgical excision is often technically very difficult due to the large masses, lack of encapsulation, and the infiltrative pattern of the fatty tissue. Unfortunately, these lipomatous depots almost always recur within the first 10 years after surgical lipectomy $[2,8,9]$. Surgical treatment should, therefore, be restricted to patients with compression of the trachea or larynx by the fat depots, or to patients with cosmetic or psychological problems. More recently, liposuction was described to replace or as an adjunct for surgical excision [2,5]. It seems to be an ideal approach to this problem in patients with masses of limited size, without scarring and with fewer complications than lipectomy. However, liposuction is often not radical enough in the submental area and it is impossible in areas where previous surgery was performed due to scar tissue. Moreover, in severe cosmetic deformities, surgical excision with removal of redundant skin is indicated. Von Hörl and Biemer [8] concluded that both approaches could be useful and had their own complications.

Although there was no history of surgery in our patients, we experienced great difficulty to aspirate enough fat tissue with our liposuction devices; however, some authors describe aspirated volumes up to 3 L [3]. In our hands, the firm constitution of this tissue prevented adequate aspiration, although we used a $5.0 \mathrm{~mm}$ canulla after adequate infiltration. Preoperative evaluation of the fat by pinch test couldn't 


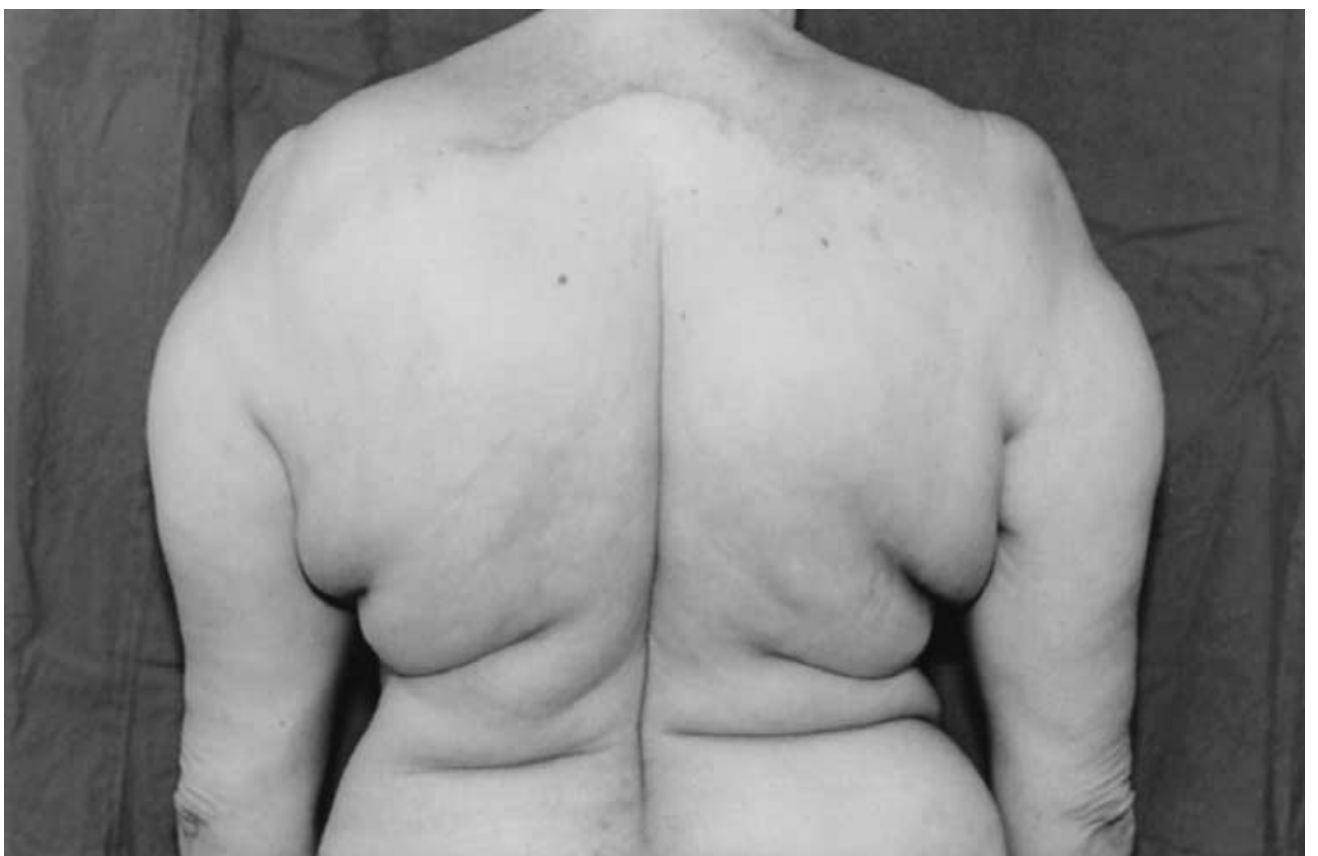

Fig. 2. Postoperative view of the $\mathrm{W}$-shaped incision at the upper aspect of the back after 8 weeks.

predict the possible failure of liposuction. On the other hand, there are no histological arguments, such as fibroplasias or other specific characteristics, that can justify the difficulties during aspiration. Faga et al. [10] performed ultrasound-assisted liposuction and concluded that the ultrasound technique allows more delicate and precise movements with less subcutaneous framework damage. However, this operation was carried out only for symptomatological relief and resulted in only a modest cosmetic result. We believe that in cosmetic indications of BSL of limited size, liposuction should be the first choice of treatment. Surgeons should be aware of the possible poor cosmetic result due to inadequate aspiration of the fat tissue. Therefore, each patient with BSL has to be informed that surgical fat excision can be necessary in case of unsatisfying liposuction results to obtain a better cosmetic result.

\section{References}

1. Argenta LC, McClatchey KD, Ferrell WJ, Newman MH: Benign symmetrical lipomatosis (Madelung's disease). Head Neck Surg 3:240, 1981
2. Carlin Marie C, Ratz JL: Multiple symmetric lipomatosis: Treatment with liposuction. J Am Acad Dermatol 18:359, 1988

3. Faga A, Valdatta LA, Thione A, Buoro M: Ultrasound assisted liposuction for the palliative treatment of Madelung's disease: A case report. Aesthet Plast Surg 25:181, 2001

4. Knopper D, Feid H, Hornberger W: Madelung's lipomatosis of the neck. Expression of an alcohol-induced disorder?. HNO 34:474, 1996

5. Ruzicka T, Vieluf D, Landthaler M, Braun-Falco O: Benign symmetric lipomatosis. J Am Acad Derm 17:663, 1987

6. Smith PD, Stadelmann WK, et al.: Benign symmetric lipomatosis (Madelung's disease). Ann Plast Surg 41:671, 1998

7. Tizian C, Berger A, Vykoupil KF: Malignant degeneration in Madelung's disease: Case report. Br J Plast Surg 36:187, 1983

8. Von Hörl C, Biemer E: Benigne symmetrische lipomatose. Lipektomie und Liposuktion in der Behandlung des Morbus Madelung. Handchir Mikrochir Plast Chir 24:39, 1992

9. Williams DW, Ginsberg LE, Moody DM, McCain BL: Madelung disease; MR findings. AJNR 14:1070, 1993

10. Wood R: Benign symmetrical lipomatosis - a complication of excessive alcohol consumption. SAMJ 77:369, 1990 